\title{
Application of Innovative Tools to Design Ergonomic Control Dashboards
}

\author{
Fabio GRANDI ${ }^{\mathrm{a}}$, Margherita PERUZZINI ${ }^{\mathrm{a}, 1}$, Claudia E. CAMPANELLA ${ }^{\mathrm{b}}$ and \\ Marcello PELLICCIARI ${ }^{\mathrm{c}}$ \\ a Dept. Engineering “Enzo Ferrari”, University of Modena and Reggio Emilia, via \\ Vivarelli 10, 41125 Modena, Italy \\ ${ }^{\mathrm{b}}$ CNH Industrial, viale delle Nazioni 55, 41122 Modena, Italy \\ ${ }^{c}$ Dept. Sciences and Method for Engineering, via Amendola 2, 42122, Reggio Emilia, \\ Italy
}

\begin{abstract}
Designing highly usable and ergonomic control dashboards is fundamental to support the user in managing and properly setting complex machines, like trains, airplanes, trucks and tractors. Contrarily, control dashboards are usually big, intrusive, full of controls and not really usable for different users. This paper focuses on the re-design of an ergonomic and compact dashboard for tractor control, proposing an innovative methodology in line with human-centered design and ergonomics principles. The study started by shifting the focus from how a machine works to how a task has to be performed and how the user interacts with the machine. It uses virtual simulations and human performance analysis tools to support the concept generation and the detailed design, and to test the new idea with users in the virtual lab. Indeed, within the virtual environment, different configurations of controls can be tested, checking which controls are mostly used and measuring human performance indexes (i.e., postural comfort and mental workload) for each configuration. Virtual mannequins can be used to as "digital twins" to interact with virtual items and to calculate robust comfort indicators during task execution. The study adopted the proposed methodology to an industrial use case to develop a usable and compact armrest for a new tractor platform. The new armrest is smaller than the previous one $(-30 \%$ in dimensions), more usable (keeping on board only frequent controls, better positioned), and more comfortable (it satisfies $95 \%$ of the population size). This new approach could be used also for the design of new products.
\end{abstract}

Keywords. Virtual simulation, Human-centered design, Human Factors, Usability

\section{Introduction}

Driving and control of agricultural machinery is a stressing activity, physically and mentally. Indeed, the use has to drive and contemporarily also execute a lot of body movements such as steering, looking forward and backward, and also control the vehicle's dashboard, using clutch, brake, control levers [1]. Recently, most tractors' producers have introduced specific dashboards where the majority of commands are located, in order to support the tractor control. However, such dashboards risk being large and invasive, full of controls and not fully adjustable to the users' preferences, as demonstrated by internal market analysis and panel tests with users. It has been also

\footnotetext{
${ }^{1}$ Corresponding Author, Mail: margherita.peruzzini@unimore.it.
} 
proved that an incorrect use of tractor commands in the long term could generate physical health problems in different upper parts of the body (i.e., arms, neck, shoulders, back, head) [2]. As a consequence, the dashboard design, including its position with respect to the seat and other interaction devices, plays an important role in determining the driver's comfort and can significantly enhance operator productivity, comfort and safety [3]. In this context, the adoption of an ergonomic approach is compulsory. It supports the inclusion of human factors in tractor design in order to respond to physical, psychological, social and cultural needs of human beings [4]. As far as industrial system design, the optimization of posture, physical overload, perceived effort, discomfort, and physical fatigue is fundamental to satisfy the users' needs, pre-vent musculoskeletal disorders and stressing conditions [5]. In this context, the analysis of human factors has a central role in the understanding of human behaviours and performance interacting with systems, and the application of that understanding to design of interactions [6].

The paper presents a set of innovative tools to re-design human-centric, ergonomic control dashboards and describes their application to a real industrial case, concerning a specific tractor dashboard re-design. In particular, the research approach integrates different methods for the acquisition of physical and physiological parameters, in order to collect the biomechanical performance of "real" users', and its virtualization within a virtual lab for simulation and testing. Indeed, the virtual environment allows to replicate the "typical" users' behaviour thanks to a "digital twin" and to test many different configurations of controls, by measuring a global comfort index for each design. Such an approach has been really usefully for the re-design the new mini armrest, based on the satisfaction of the users' needs.

\section{Research Background}

The goal of ergonomics is not only to improve work performance but also to improve human comfort as well as safety [4]. If ergonomic aspects are not given due consideration, the performance of the system will be poor and the effective working time will be reduced. Moreover, ergonomics is a typically a transdisciplinary discipline since it requires technical as well as social-science skills and certainly involves people from practice. Technical science concerns with the design of machines, interfaces and information system. Social science assists in identifying the needs of users in order design usable and useful interfaces and interaction systems. Application of ergonomics to industrial systems is definitely transdisciplinary as it requires multiple disciplines, as well as people from practice, because the problems encountered can be multi-faceted [7].

The best approach to ergonomics is the preventive one, which consists of anticipating ergonomics problems during the design stage in order to optimize the overall system design to conform to the physiological, psychological, and behavioural capabilities of workers. There are many factors acting as stress on the operator during the work. These stresses may be due not only to physical workload and uncomfortable postures, but also to task complexity, overload of information to be handled, time pressure [8]. This fatigue can be measured in terms of strain on the tractor driver [9]. The most common analyses typically include electrocardiography (for heart rate monitoring), electromyography (for monitoring muscles activity through their electrical potentials), the pneumography (for respiration control) or the skin conductivity (to measure sweat activity) [10-11]. The multimodal dimension of stress makes the research field very broad; however, four main criteria can be distinguished in detecting the human stress, 
according to ISO 10075-3 [12]: psychological, physiological, behavioural, and biochemical. From a physiological point of view, the increase of nervous system activity changes hormone levels in the body and provokes reactions such as sweat production, increased heart rate and muscle activation. Breathing becomes faster and increases blood pressure. Usually skin temperature and HRV fall. The diameter of the pupils can vary. Finally, behavioural reactions include eye movements and eye change rates, as well as changes in facial appearance and head movements.

There are many physiological signals to be used in stress detection and some of them have shown to provide reliable information about peoples' real-time stress levels [13]. Electrocardiogram (ECG) is one of the most used signals in stress detection research because it reflects directly the activity of the heart, mainly the Heart Rate (HR, defined as the number of heart beats per minute), the Heart Rate Variability (HRV, defined as the temporal variation between sequences of consecutive heart beats) and the LF/HF ratio (low frequency / high frequency). Moreover, eye activity in terms of number of gazes and blink rates can be measured with infrared eye tracking systems or with image processing techniques applied to visual spectrum images of the eyes. Thus, pupil dilatation exhibits changes under stress situations and can be measured by the dilation mean value, standard deviation, gaze spatial distribution, number of fixations, as well as the blink rate or blinking frequency.

\section{Research Approach}

The research approach integrates different tools for the acquisition of physical and physiological parameters in order to objectify the users' experience and workload, as follows:

- Use of sensors for real-time analyses of the main physiological parameters, which can provide a clear feedback on the driver's state without interference with the driver's activities. The adopted sensors refer to: a bio-sensor for electrocardiography (for heart rate (HR) and heart rate variability (HRV) monitoring), breathing monitoring (for breath rate (BR) analysis) (i.e., BH3 by Zephyr) and an eye-tracking device for eyes' fixation analysis and visual attention mapping (i.e., Glasses 3 pro by Tobii);

- Motion capture for real-time analyses of body movements, to measure the position of the different body parts (e.g., arms, hands, head) and to objectify the human interaction in terms of distance, joint angles, instantaneous speed or acceleration) (i.e., Xsens MVN by Xsens);

- A human simulation software for virtualization of human-machine interaction and physical ergonomic analysis by Dreyfuss methods (i.e., Jack by Siemens);

- A CAN (Controller Area Network) data collection for analyses of the human inter-action with the control devices and interface, to check whether and what type of interaction is taking place during task execution.

Moreover, the research approach is based on a three-phase experimental study.

Phase 1. First of all, real users are monitored during field tests interacting on the real, physical dashboard using the proposed set of tools, in order to define the typical users' actions and to identify the main critical tasks and the main interaction difficulties. During this phase, a sample of users, representative of the target users of the specific product under investigation, drives a real tractor executing a set of pre-defined activities, and their physiological and physical data are collected by wearable technologies 
(BioSensor BH3 and eye-tracking Glass 2). In the meanwhile, interaction data from the CAN network are collected from the tractor itself. This phase allows to define the users' needs and to analyse how commands and controls are used, in order to highlight ergonomic issues and propose a more usable grouping for the new dashboard design.

Phase 2. On the basis of the data collected during Phase 1, different dashboard designs are produced and prototyped. After that, simulation sessions within a Virtual Lab involving users are organized; users are virtualized and a virtual mannequin is associated to each user to create the specific digital twin. Different cabin layouts and dashboards design are simulated and verified on the basis of virtual mannequins generated by tests with users. Virtual mannequins are used to represent different-sized operators, and for each of them to check the visibility, reachability and joint comfort of humans on dashboard proposals.

Phase 3. Finally, the motion capture system (Xsens) is used to track real users' movements during virtual testing in Lab and emulate the human-machine interaction within an immersive environment. The immersive simulation is useful to evaluate he interaction with the final dashboard layout and to validate it by expert-based assessment. Moreover, motion capture allows to create more reliable digital twins of real users, and to effectively measure the joint angles and comfort indexes. Real missions can be simulated and the new dashboard design validated in the virtual environment.

Fig. 1 shows the adopted tools and the data elaboration framework.

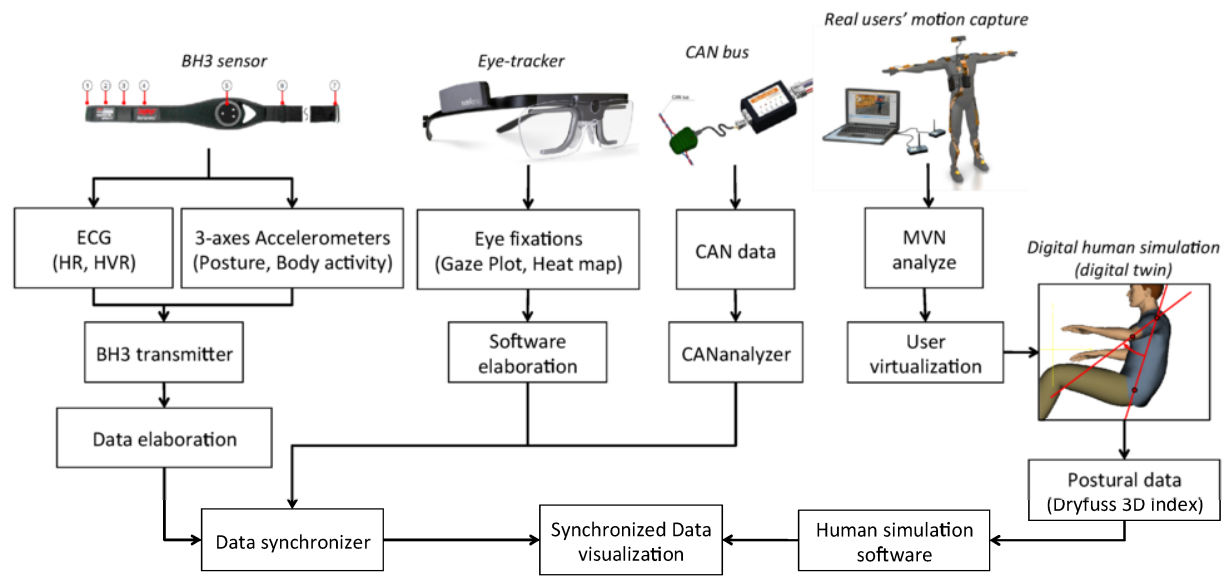

Figure 1. The research data elaboration framework.

\section{Experimental testing on the industrial use case}

\subsection{The industrial use case}

The use case focuses on the Steyr brand, in particular on the re-design of the control dashboard of the Expert CVT model. This tractor is aimed at customers who need high performance tractor with compact dimensions and high comfort. This model is presented on the market for its particular ergonomic character and its multi-controller armrest able to merge comfort, flexibility and high-quality performance. Indeed, its cabin is characterized by smaller dimensions (minor length and width with respect to other Steyr 
models), lower intrusiveness for the operator trunk-legs, and improved sensation of roominess inside the cabin. Moreover, it has an innovative controls layout, with many controls managed with push-buttons, grouped in a more organized-intuitive way using different panels to help operators to immediately find each control. Both characteristics improve the human comfort during working in a tractor cabin and reduce the mental workload.

The proposed approach was applied for the re-design of the Expert CVT armrest. For Phase 1, five "typical" users were selected and involved in on-field testing. It was useful to map the users' behaviours and needs. After that, Phase 2 was oriented to link the virtual mannequins belonged to different percentiles $(5 p, 50 p$ and $95 p)$ were used to represent different-sized operators, and for each of them to check the visibility, reachability and joint comfort of humans on cabin armrest proposals. Many proposals were investigated, especially to find out the good compromise between reduced dimension of the armrest and a visible and effective control layout to be operated in comfort for different human percentiles. Fig. 2 shows the virtual prototype of the Steyr Expert CVT armrest and examples of virtual tests with mannequins belonged to different percentiles (5p, 50p and 95p). Fig. 3 shows examples of virtual testing about reachability and visibility.
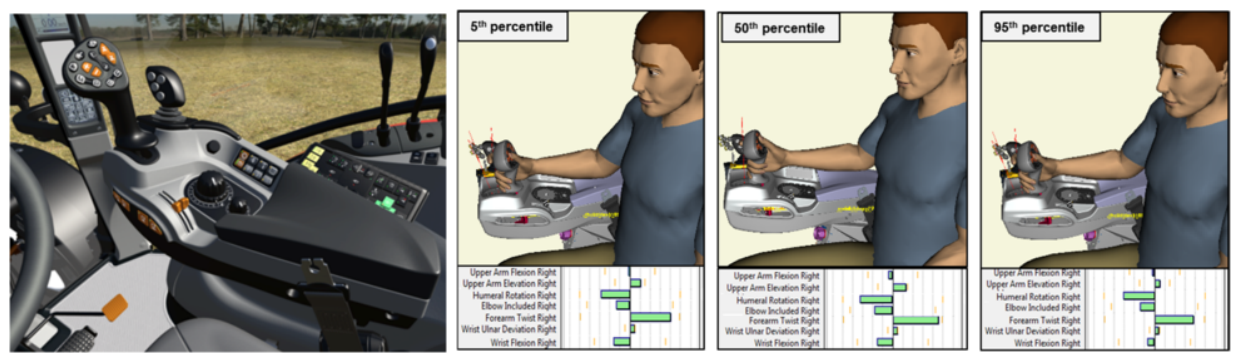

Figure 2. Virtual prototype of the Steyr Expert CVT armrest (left) and virtual tests on different percentiles $5 \mathrm{p}, 50 \mathrm{p}$ and $95 \mathrm{p}$ (right).
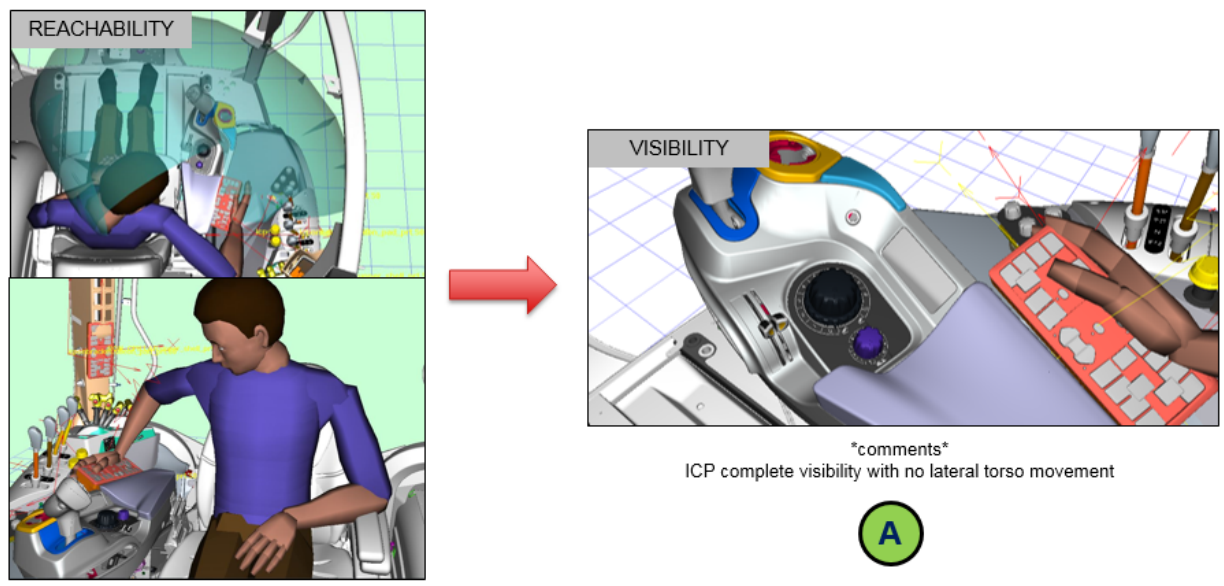

ICP complete visibility with no lateral torso movement

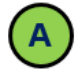

Figure 3. Examples of virtual testing checking reachability and visibility on different layouts.

After that, a new armrest was designed to improve the user comfort and armrest usability. Finally, Phase 3 focuses on the simulation in Virtual Lab using the motion 
capture and real users' involvement to check the interaction modalities and improved performance. Fig. 4 shows the new armrest layout and immersive testing in Virtual Lab.
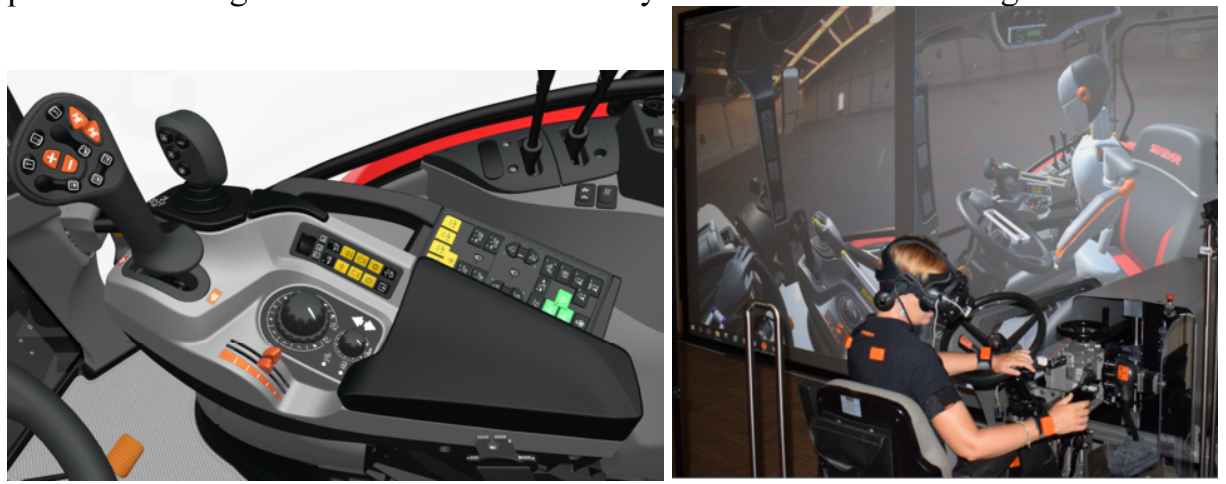

Figure 4. New armrest layout based on intuitive-grouped control panels (left) and immersive tests in Virtual Lab (right).

\subsection{Results and discussion}

During experimental testing, data about the user physiological and physical state were collected and correlated with the interface use and the visual interaction quality. After that, data synthesis and post-processing allowed analysing the user workload in order to understand the level of comfort and the usability of the armrest, considering also the level of stress and the perceived quality of interaction. The main advantages of the proposed approach are the use of virtual users during the design phases, in order to validate different design solutions, and the limitation of the involvement of real users only to the last phase. Immersive virtual simulation allowed creating a digital environment where users can interact in advance with the product features, to address the main interaction criticalities during the design stage. Predictive analysis can be carried out before real product realization, and an optimized product was created, avoiding late optimization actions. Indeed, the use of digital simulations allowed easily comparing different product layouts, replicating the sequence of actions, predicting the user movements, and defining the best design solution.

The main results obtained by the new armrest design are:

- Higher comfort index for all users, and in particular for taller users (95p) as demonstrated by the Abita4t maps toolkit. Fig. 5a highlights how 5p, $50 p$ and $95 p$ manikins can comfortably accommodate in the new armrest cushion, using all armrest possible adjustments (all green boxes, higher than $75 \%$ ). Fig. 5b shows seat comfort maps;

- $\quad$ Reduced encumbrance (the new armrest is 20\% shorter and 30\% thinner than the previous one). Fig. 6 shows its main dimensions and compared it with the previous armrest;

- Higher performance and reduced time for tractor control due to an optimized control layout with push button panels (indeed, the new armrest allows keeping on board only frequent controls positioned in ergonomic way). 
a)
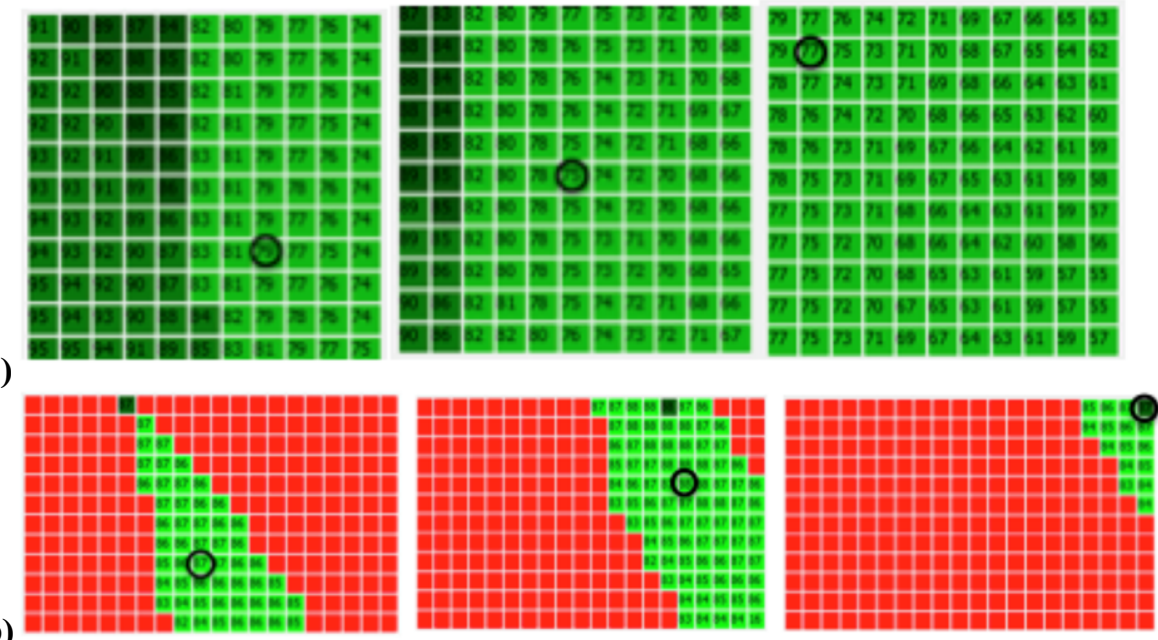

Figure 5. Comfort indices (a) and seat maps comfort indices (b) for the new armrest design (5p, 50p, 95p).

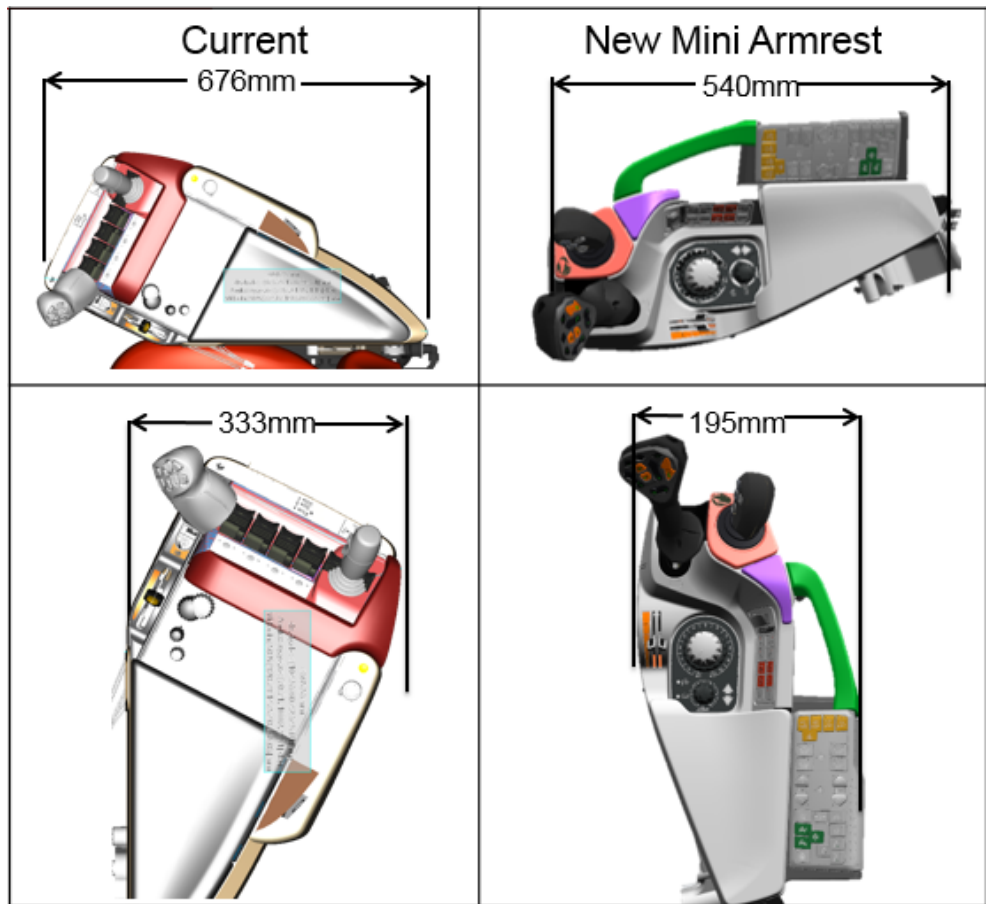

Figure 6. Dimension comparison between the previous armrest and new compact-ergonomic armrests.

\section{Conclusions}

This paper presented a set of innovative tools to support ergonomic, human-centered design of control dashboards and described its application to the re-design of control board of tractors, in collaboration with $\mathrm{CNH}$ Industrial. The new approach combines 
digital technologies, virtual reality and human monitoring devices to objectify the user experience and to introduce ergonomics validation before product realization. The new approach allowed to reduce re-design time, to reduce the final product costs, and to improve the user comfort as well as dashboard usability. In particular, the new armrest is smaller than the previous one (-30\% in dimensions), more usable (keeping on board only frequent controls, better positioned), and more comfortable (it satisfies $95 \%$ of the population size). Such results could be used also to guide the re-design for other tractors controls and dashboards, so that human comfort can be improved and any task can be felt as natural as possible, encouraging good posture and safe behaviours, and reducing cost of prototypes and time to market. From a societal viewpoint, the inclusion of human factors in systems design can overcome the current issues due to changes in technologies and requirements of workers, and enhance their work conditions. The proposed methodology merges different disciplines in order to assess the general comfort of the user, according to a transdisciplinary approach.

\section{Acknowledgement}

The authors wish to acknowledge CNH Industrial staff for the precious collaboration.

\section{References}

[1] B. Bashiri, D.D. Mann, Automation and the situation awareness of drivers in agricultural semi-autonomous vehicles. Biosystems Engineering, vol. 124, 2014, pp. 8-15.

[2] C.R. Metha, V.K. Tewari, Seating discomfort for tractor operators - A critical review, International Journal of Industrial Ergonomics, 2000, vol. 25(6), pp. 661-674.

[3] H. Hsiao, J. Whitestone, B. Bradtmiller, R. Whisler, J. Zwiener, C. Lafferty, T.Y. Kau, M. Gross, Anthropometric criteria for the design of tractor cabs and protection frames, Ergonomics, vol. 48 (4), 2005, pp. 323-353.

[4] N.N., Ergonomics of human system interaction - Part 210: Human-centered design for interactive systems, International Organization for Standardization (ISO), ISO 9241-210, 2009

[5] S. Pheasant, Body Space: Anthropometry, Ergonomics and the Design of Work, Taylor \& Francis, Philadelphia, pp. 121-123, 1999.

[6] J.R. Wilson, Fundamentals of ergonomics in theory and practice, Applied Ergonomics, vol. 31, 2000, pp. 557-567.

[7] N. Wognum, C. Bil, F. Elgh, M. Peruzzini, J. Stjepandić, Transdisciplinary systems engineering: implications, challenges and research agenda, Int. J. Agile Systems and Management, 2019, Vol. 12 (1), pp. 58-89.

[8] J.Y. Zhang, W.W. Qiu, H.J. Fu, M.T. Zhang, Q.G. Ma, Review of techniques for driver fatigue detection, Applied Mechanics and Materials, 2013, vols. 433-435, pp. 928-931.

[9] M. Peruzzini, F. Grandi, M. Pellicciari, C.E. Campanella, User Experience Analysis Based on Physiological Data Monitoring and Mixed Prototyping to Support Human-Centred Product Design. In: F. Rebelo, M. Soares (eds) Advances in Intelligent Systems and Computing, 7vol. 77, 2019, pp. 401-412.

[10] K. Boubaker, A. Colantoni, E. Allegrini, L. Longo, S. Di Giacinto, D. Monarca, A model for musculoskeletal disorder-related fatigue in upper limb manipulation during industrial vegetables sorting, International Journal of Industrial Ergonomics, 2014, vol. 44, pp. 601-605.

[11] J.A. Healey, R.W. Picard, Detecting Stress During Real-World Driving Tasks Using Physiological Sensors, IEEE Transactions on intelligent transportation systems, 2005, vol. 6, pp. 156-166.

[12] N.N., Ergonomic principles related to mental workload - Part 3: Principles and requirements concerning methods for measuring and assessing mental workload, International Organization for Standardization (ISO), ISO 10075-3, 2005.

[13] A. Alberdi, A. Aztiria, A. Basarab, Towards an automatic early stress recognition system for office environments based on multimodal measurements: A review, Journal of Biomedical Informatics, 2016, vol. 59, pp. 49-75. 